\title{
An Unusual Presentation of Jejunal Atresia in a Toddler
}

Rebecca AbelI* ${ }^{1 *}$ Julie Khlevner ${ }^{1}$, Thomas Lee ${ }^{2}$ and Anupama Chawla1

${ }^{1}$ Division of Gastroenterology, Hepatology and Nutrition, Stony Brook Long Island Children's Hospital, USA

${ }^{2}$ Division of Pediatric Surgery, Stony Brook Long Island Children's Hospital, USA

\begin{abstract}
Intestinal atresia is a well recognized cause of bowel obstruction in the neonatal period. Reports on the incidence and symptoms of intestinal atresia presenting outside the neonatal period are scant. We report an 18 month old previously healthy female who presented with a four day history of tarry stools, with associated fatigue. Lab work revealed a hemoglobin of 4 , hematocrit of 13 , and reticulocyte count of 6.8 suggesting an acute bleed. She was admitted to the PICU and transfused. An upper endoscopy was performed which demonstrated copious amounts of undigested food in the distal duodenum. No active sources of bleeding were located and the scope could not be passed beyond this point. Upon repeat endoscopy 5days later, after an appropriate bowel regimen, multiple ulcerated lesions were seen in the proximal jejunum at an area of narrowing through which a $5.9 \mathrm{~mm}$ infant scope was not able to be passed. The patient was taken to surgery and the small bowel was found to be malrotated. A discrepancy in the bowel was identified at the proximal jejunum and an $8 \mathrm{~cm}$ length of bowel was resected. The patient did well post-operatively. The overall presentation of this case is unusual in many ways, from age at onset to atypical clinical symptoms. In conclusion, intestinal atresia should be part of the differential diagnosis in children outside of the neonatal period presenting with upper gastrointestinal bleed, requiring surgical intervention.
\end{abstract}

Keywords: Jejunal Atresia; Endoscopy; Anemia; Surgical Resection; Pediatric

\section{Introduction}

Intestinal atresia is a well recognized cause of bowel obstruction in the neonatal period. Reports on the incidence and symptoms of intestinal atresia presenting outside the neonatal period are scant. These conditions may involve any portion of the gastrointestinal tract, the small bowel being the most commonly affected [1]. Of the affected small bowel, jejunoileal atresia, with obstruction of jejunum or ileum, is quite rare with few reported cases. It historically has been categorized into Type I (mucosal web), Type II (atretic fibrous cord), Type IIIa (V shaped mesenteric defect), Type IIIb (apple peel atresia) and type IV (multiple atresias) [2]. Common presentations and clinical findings in the newborn include recurrent bilious emesis, abdominal distention, jaundice, and failure to pass meconium [1]. Symptoms in a child may include failure to thrive in addition to recurrent bilious emesis. Treatment usually involves surgical resection of the area of obstruction although newer options have become available in recent years such as endoscopic balloon dilatation [3]. We report an unusual case of jejunal atresia in an 18 month old who presented with severe anemia and tarry stools as the only symptoms.

\section{Case Report}

An 18 month old previously healthy female presented with a four day history of tarry stools, with associated fatigue. Review of systems was negative, including no history of vomiting or weight loss. Her weight was $9.2 \mathrm{~kg} \mathrm{(3 \% )} \mathrm{and} \mathrm{height} \mathrm{was} 76 \mathrm{~cm}(7 \%)$. She was tachycardic with a heart rate of 142 , other vital signs were normal for age. On physical examination, she appeared pale. Abdomen was soft, non-tender, and non-distended. Labwork revealed a hemoglobin of 4 , hematocrit of 13 , and reticulocyte count of 6.8 suggesting an acute bleed. Comprehensive metabolic panel was within normal limits. Stool guaiac was positive. Helicobacter pylori stool antigen was negative. Upright and flat abdominal radiograph demonstrated nononbstructive gas pattern with no evidence of dilated loops of bowel. A Meckel's diverticulum scan was negative. She was admitted to the PICU and transfused $10 \mathrm{cc} / \mathrm{kg}$ of packed red blood cells with an increase in her hemoglobin to 8.2.
An upper endoscopy was performed which demonstrated copious amounts of undigested food in the distal duodenum. Despite flushing of the area and removal of some of the food with the help of Roth net and rat tooth, significant amount of food remained. No active sources of bleeding were located and the scope could not be passed beyond this point. The patient was given polyethylene glycol 3350 to aid in progression of the undigested food so that the area could be better visualized. Upon repeat endoscopy 5days later, multiple ulcerated lesions were seen in the proximal jejunum at an area of narrowing through which a $5.9 \mathrm{~mm}$ infant scope was not able to be passed. There was concern for stenosis and surgery was consulted.

In the operating room, the patient's small bowel was found to be malrotated with the cecum and ascending colon at midline. A discrepancy in the bowel was identified at the proximal jejunum with a bulbous shape proximally that appeared chronically dilated with smaller jejunal lumen distally. An $8 \mathrm{~cm}$ length of proximal jejunum was resected with primary end to end anastamosis. Pathology confirmed that there was a marked difference in diameter between the opposite ends of the resected jejunal segment. The diameter of the distal end measured $2.0 \mathrm{~cm}$ and diameter of the proximal end measured $5.5 \mathrm{~cm}$. Areas of acute and chronic inflammation, mural edema and additional reactive changes were reported in the proximal dilated jejunum where ulcerations were visualized. The patient did well post-operatively.

\section{Discussion}

Incidence of jejunal atresia outside of the neonatal period is

*Corresponding author: Rebecca Abell, DO, Stony Brook Long Island Children's Hospital, Division of Pediatric Gastroenterology, Hepatology, and Nutrition, HSC Level 11 Rm 080, Stony Brook, NY 11794, USA, Tel: 1-631-444-8115; Fax: 1-631 444-6045; E-mail: rebecca.abell@sbumed.org

Received May 22, 2012; Published July 26, 2012

Citation: Abell R, Khlevner J, Lee T, Chawla A (2012) An Unusual Presentation of Jejunal Atresia in a Toddler. 1: 112. doi:10.4172/scientificreports.112

Copyright: (C) 2012 Abell R, et al. This is an open-access article distributed unde the terms of the Creative Commons Attribution License, which permits unrestricted use, distribution, and reproduction in any medium, provided the original author and source are credited. 
extremely rare. There are very few reported cases in the literature all of which presented with recurrent emesis. A study by Vecchia et al. [1] looked at intestinal atresias over 25 years at their institution. Out of 277 reported cases, 128 were jejunoileal. Of those, $23 \%$ were classified as type I, $27 \%$ as type II, $18 \%$ as type IIIa, $7 \%$ as type IIIb, and $24 \%$ as type IV. It is believed that a late intrauterine vascular insult is the cause of most cases [4].

Common presentation of jejunal atresia beyond the neonatal period is bilious vomiting, abdominal distention, and failure to thrive. Gastroschisis, omphalocoele, and cystic fibrosis have also been reported to be associated with this condition [4]. Our case is unusual in that the infant had an unremarkable perinatal history, no reported history of poor weight gain, and did not present with vomiting or abdominal distention. Tarry stools and anemia as the presenting symptoms in our 18 month old, likely contributed to the delay in diagnosis of the jejunal atresia.

Diagnosis of jejunal atresia is made with the use of an abdominal x-ray which commonly demonstrates dilated loops of bowel, often associated with air/fluid levels [2]. An upper gastrointestinal series can be helpful to localize the area of narrowing and screen for other congenital anomalies such as malrotation, concurrent duodenal and colonic atresias [1].

Management of jejunal atresia, typically is surgical, with excision of the affected area. Intraoperative findings that have been found with jejunal atresia are volvulus, malrotation, meconium peritonitis, meckel diverticulum, and intussusceptions [1]. Our patient was found to have malrotation, but did not have clinical symptoms of obstruction on presentation. However, with the advancement of endoscopic technology and skills, endoscopic balloon dilatation may be an effective treatment in the management of a case such as this [3].

Our case is interesting in that an upper endoscopy was warranted to look for a source of an upper gastrointestinal bleed, allowing us to discover the ulcerations at the area of narrowing. The ulcerations were likely secondary to chronic stasis of undigested food with bacterial overgrowth. Significant bleeding of the ulcers led to presentation of tarry stools and severe anemia.

The overall presentation of this case is unusual in many ways, from age at onset to atypical clinical symptoms. In conclusion, intestinal atresia should be part of the differential diagnosis in children outside of the neonatal period presenting with upper gastrointestinal bleed.

\section{References}

1. Dalla Vecchia LK, Grosfeld JL, West KW, Rescorla FJ, Scherer LR, et al. (1998) Intestinal atresia and stenosis: a 25-year experience with 277 cases. Arch Surg 133: $490-496$.

2. Kleinman RE, Goulet OJ, Mieli-Vergani G (2008) Walker's Pediatric Gastrointestinal Disease, Edition 5, Vol1, 223-226.

3. Mochizuki K, Obatake M, Kosaka T, Tokunaga T, Eguchi S, et al. (2011) Endoscopic balloon dilatation for congenital membranous stenosis in the jejunum in an infant. Pediatr Surg Int 27: 91-93.

4. Seltz LB (2008) Case 1: A green case of failure to thrive. Paediatr Child Health 13: 685-687. 